\title{
Surface EMG of jaw elevator muscles: effect of electrode location and inter-electrode distance
}

\author{
T. CASTROFLORIO*, D. FARINA ${ }^{\dagger}$, A. BOTTIN ${ }^{\dagger}$, M. G. PIANCINO*, P. BRACCO* $\delta$ \\ R. MERLETTI ${ }^{\dagger}{ }^{*}$ Specialization School of Orthodontics, Department of Biomedical Sciences and Human Oncology, Università di \\ Torino, Torino, Italy and ${ }^{\dagger}$ Centro di Bioingegneria, Dip. di Elettronica, Politecnico di Torino, Torino, Italy
}

SUMMARY This study addresses methodological issues on surface electromyographic (EMG) signal recording from jaw elevator muscles. The aims were (i) to investigate the sensitivity to electrode displacements of amplitude and spectral surface EMG variables, (ii) to analyse if this sensitivity is affected by the inter-electrode distance of the bipolar recording, and (iii) to investigate the effect of interelectrode distance on the estimated amplitude and spectral EMG variables. The superficial masseter and anterior temporalis muscles of 13 subjects were investigated by means of a linear electrode array. The percentage difference in EMG variable estimates from signals detected at different locations over the muscle was larger than $100 \%$ of the estimated value. Increasing the inter-electrode distance resulted in a significant reduction of the estimation variability because of electrode displacement. A criterion for electrode placement selection is suggested, with which the sensitivity of EMG variables to small electrode displacements was of the order of $2 \%$ for spectral and $6 \%$ for amplitude variables. Finally, spectral and, in particular, amplitude EMG variables were very sensitive to inter-electrode distance, which thus should be fixed when subjects or muscles are compared in the same or different experimental conditions.

KEYWORDS: surface electromyography, linear electrode arrays, electrode location, jaw elevator muscles

Accepted for publication 1 July 2004

\section{Introduction}

Surface electromyography (EMG) provides non-invasively information on muscle properties. Modelling studies indicate that electrode location over the muscle has a large influence on the characteristics of the recorded EMG signals (1-4). For example, surface EMG features are sensitive to small electrode displacements if the detection point is close to the innervation zones or tendons (5-9). Thus, comparison of results obtained in different days with replacement of the electrodes may be critical. These issues have been investigated in modelling studies $(5,6)$ and experimental works (7-9), especially for muscles with simple architecture.

Many surface EMG studies on the masticatory muscles were performed in the past [see, for example Refs
(10-14)]. However, for these muscles, there have been few works addressing methodological issues of the surface EMG detection $(15,16)$. In most studies, the electrodes were placed on the muscle belly without indications of specific points along the muscle length and the inter-electrode distance was usually different among different studies.

For jaw elevator muscles, there is a lack in the literature on quantitative indications on the sensitivity of variables extracted from the surface EMG on the type of recording (location of the electrodes, inter-electrode distance). These issues are important for the repeatability of the results and the possibility of comparing data from different studies.

In this work we apply the multi-channel surface EMG technique for the investigation of the myoelec- 
tric activity of jaw elevator muscles. Placing many electrodes over the muscles, instead of the classic single bipolar recordings, allows to assess the myoelectric activity at different muscle locations. The study is mainly methodological with direct implications on the application of the surface EMG approach for muscle characterization in both basic research and clinical studies. The work is performed on the superficial masseter and the anterior temporalis muscles. The aims of the work were 1) to investigate the sensitivity of amplitude and spectral surface EMG variables to small electrode displacements, 2) to analyse if this sensitivity is affected by the interelectrode distance of the bipolar recording, and 3) to investigate the effect of inter-electrode distance on the estimated amplitude and spectral EMG variables.

\section{Materials and methods}

\section{Subjects}

A sample of 13 healthy subjects [nine males, four females, age, mean $\pm \mathrm{SD}, 27 \cdot 3 \pm 2 \cdot 7$ years, overjet $3 \pm 1 \mathrm{~mm}$, overbite $3 \pm 1, \mathrm{SpP} \wedge \mathrm{GoGn} 22 \pm 5^{\circ}$ - mesomorphic craniofacial typology according to Bracco et al. $(17,18)]$, was selected from a group of 38 students and voluntary assistants of the Orthodontics School of the University of Torino, Torino, Italy. Inclusion criteria were 1) no signs or symptoms of temporomandibular disorders, 2) no cross-bite, 3) no orthodontic treatment, 4) no prosthetic rehabilitation, and 5) no missing teeth (with the exception of the third molars). All subjects participated to the experiment after giving written informed consent. The study was approved by the local Ethics Committee.

\section{Experimental protocol}

The subject sat on a comfortable chair without head support, with the trunk in an erect posture and natural head position. The eyes were fixed on a target in front of him/her, $1 \mathrm{~m}$ away. The muscles investigated were the masseter, superficial bundle, and anterior temporalis of the right and left side. For each muscle, the subject was asked to produce six maximum voluntary clenching in the intercuspal position sustained for $10 \mathrm{~s}$. A rest period of $2 \mathrm{~min}$ was given between each clenching.

\section{Surface EMG signal recording}

Surface EMG signals were detected by a linear array of 16 electrodes $(19,20)$ (silver point electrodes, $1 \mathrm{~mm}$ diameter, $2.5 \mathrm{~mm}$ inter-electrode distance between centres). The superficial bundle of the masseter was investigated with the array placed on the mandibular angle - cantus straight line, with the last electrode corresponding to the mandibular angle (Fig. 1). The gonion-cantus distance was $100 \cdot 76 \pm 8 \cdot 86 \mathrm{~mm}$. The electrode locations over the muscle will be specified in the following as the distance from the mandibular angle, normalized with respect to the mandibular angle - cantus distance (and reported in percentage).

Two reference lines were considered for the noninvasive assessment of the anterior temporalis muscle. The first was the straight line passing between the mandibular angle and condylar head, rotated forward with an inclination of $20^{\circ}$. The second was the line tangent to the auricle ear and passing through the cantus. The array was placed along the first line with the last electrode on the crossing between the two reference lines (Fig. 1). The electrode location over the anterior temporalis will be indicated in the following as the distance from the crossing of the two selected lines (in absolute and not percentage values).

The array was fixed on the skin with adhesive tape. The portion of each muscle covered by the surface electrodes was $37.5 \mathrm{~mm}$ (16 electrodes with $2.5 \mathrm{~mm}$ inter-electrode distance). The skin was cleaned and slightly abraded with abrasive paste (Every, MEDITEC, Parma, Italy) before electrode placement.

The signals were detected in single differential mode to minimise power line interference, amplified (16-channel surface EMG amplifier, EMG 16, LISiN; Prima Biomedical \& Sport, Treviso, Italy), filtered ( $3 \mathrm{~dB}$ bandwidth, 10-500 Hz), sampled at $2048 \mathrm{~Hz}$, and converted to a numerical format using a 12-bit A/D converter. The single differential detection was performed between adjacent electrodes resulting in an inter-electrode distance of $2.5 \mathrm{~mm}$.

\section{EMG signal analysis}

By summation of consecutive single differential signals detected with the array, it was possible to derive bipolar recordings as acquired with inter-electrode distances multiple of $2.5 \mathrm{~mm}$ [for details refer to Farina et al. 
Fig. 1. (a) Schematic representation of the anatomical lines used for electrode location and examples of surface multi-channel EMG signals detected from the anterior temporalis muscle of one subject (right side). (b) Representative signals detected from the anterior temporalis muscle. The signals resulting from three inter-electrode distances are shown. Note the different amplitude scale in the three cases. The location of the innervation zones of a few potentials is indicated for the recordings with 2.5-mm inter-electrode distance. A.U. stands for arbitrary units. (a)
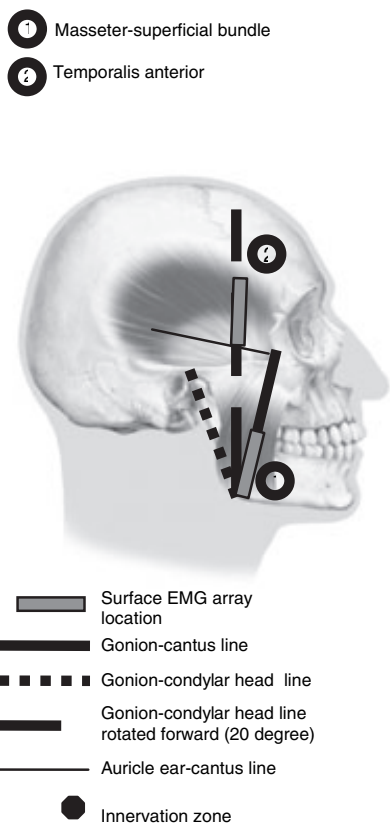

(b) Array upper part $2.5 \mathrm{~mm}$ interelectrode distance
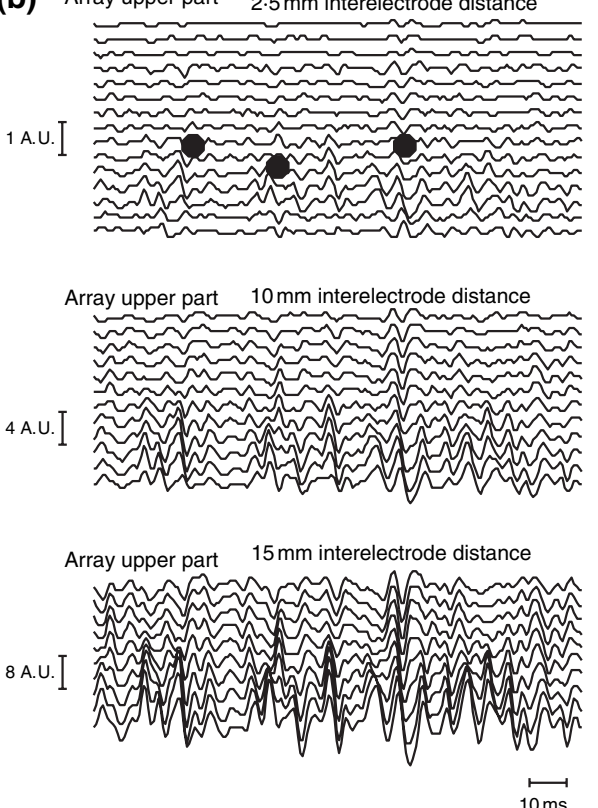

(21)]. Thus, signals detected with inter-electrode distance in the range $2.5-20 \mathrm{~mm}(2.5 \mathrm{~mm}$ increments) were obtained and analysed (eight inter-electrode distances in total).

The average rectified value (ARV) and mean power spectral frequency (MNF) were calculated from the recorded signals for the eight inter-electrode distances and for all electrode locations (22). These variables were computed from non-overlapping, adjacent signal epochs of $1 \mathrm{~s}$, resulting in 10 estimates for the 10-s long maximal contractions performed. The regression line of these estimates was computed and its initial value (at time $t=0$ ) was used for further analysis.

The sensitivity to electrode displacements was assessed by two indexes of variability of EMG estimates with electrode location (21). The total variability $\Delta_{\text {tot }}$ was defined, for each EMG variable, as the difference between the highest and the lowest variable value estimated over all electrode locations and normalized with respect to the mean value of the variable over the same locations. $\Delta_{\text {tot }}$ was computed for the eight interelectrode distances. This index indicates the largest difference (as percentage of the mean) which can occur between estimates of an EMG variable when the electrodes are placed without specific criteria on the muscle region covered by the array.

The minimum variability $\Delta_{\min }$ was defined, for each EMG variable, as the minimal percentage difference among all the percentage differences in EMG variable estimates from adjacent electrode locations. Thus, it indicates the difference which may occur in EMG variable estimates when the recording system is placed in the location with minimal sensitivity to small electrode displacements with a possible error of $\pm 2.5 \mathrm{~mm}$ (the original inter-electrode distance). The location resulting in $\Delta_{\min }$ for $2.5 \mathrm{~mm}$ inter-electrode distance was considered as the optimal one.

\section{Statistical analysis}

The data were analysed using three-way repeated measurements analysis of variance (ANOVA), followed by post hoc Student-Newman-Keuls (SNK) pair-wise comparison, when required. Statistical significance was set to $P<0 \cdot 05$. Data are reported as mean and standard deviation (s.d.) or mean and standard error of the mean (s.e.), as specified in the text.

\section{Results}

Maximal variability of surface EMG variables in the muscle portion investigated

Figure 2a shows $\Delta_{\text {tot }}$ of ARV and MNF as a function of the inter-electrode distance for the two muscles investigated. ARV showed higher sensitivity to location than 

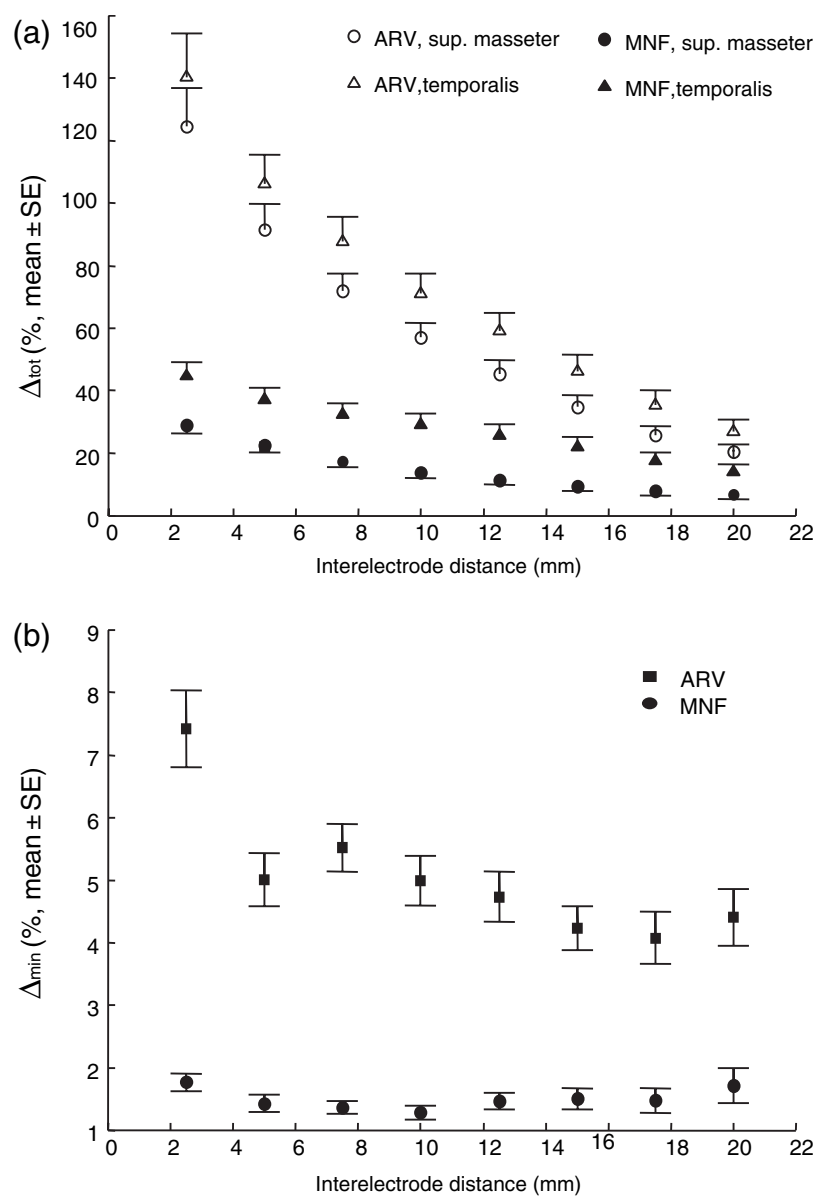

Fig. 2. (a) ARV and MNF $\Delta_{\text {tot }}$ as a function of inter-electrode distance for the two muscles investigated. Both sides are pooled together as the side is not a factor influencing $\Delta_{\text {tot }}$ (see text for the statistical analysis). b) ARV and MNF $\Delta_{\text {min }}$ as a function of the inter-electrode distance. The results from the two muscles and both sides are pooled together as neither the muscle nor the side are factors significantly influencing $\Delta_{\min }$ (see text for the statistical analysis). MNF $\Delta_{\min }$ is not influenced by inter-electrode distance but it is reported as a function of the inter-electrode distance for comparison with ARV.

MNF; $\Delta_{\text {tot }}$ reached values higher than $100 \%$ for small inter-electrode distances and values near $40 \%$ for frequently used inter-electrode distances.

A three-way (factors: muscle, side, inter-electrode distance) ANOva of MNF $\Delta_{\text {tot }}$ revealed significant dependency on the muscle $(F=9.21, P<0.01)$ and inter-electrode distance $(F=117 \cdot 06, P \ll 0 \cdot 001)$. The post hoc SNK test disclosed pair-wise differences $(P<0.01)$ between the temporalis and masseter muscles (Fig. 2a). Moreover, the post hoc SNK test disclosed differences between all the inter-electrode distances $(P<0 \cdot 05)$.
The $\Delta_{\text {tot }}$ of ARV also depended (three-way ANovA with factors muscle, side, and inter-electrode distance) on the muscle and inter-electrode distance $(F=3.73$, $P<0.05 ; \quad F=130 \cdot 16, \quad P \ll 0.001$, respectively), with the temporalis muscle showing significantly larger values of $\Delta_{\text {tot }}$ than the masseter muscle (SNK test, $P<0.05$ ). ARV $\Delta_{\text {tot }}$ was different (pair-wise comparisons by SNK test) for all the inter-electrode distances $(P<0.05)$, except between $17.5 \mathrm{~mm}$ and $20 \mathrm{~mm}$.

\section{Minimal variability of EMG variables to electrode displacements}

Figure $2 \mathrm{~b}$ reports $\Delta_{\min }$ of ARV and MNF. As for $\Delta_{\text {tot }}, \Delta_{\text {min }}$ is smaller for the frequency than for the amplitude variable. $\Delta_{\min }$ depends on inter-electrode distance but to a less extent than $\Delta_{\text {tot }}$.

A three-way (factors: muscle, side, and inter-electrode distance) ANOva of MNF $\Delta_{\text {min }}$ was not significant for any of the factors considered (Fig. 3). On the contrary, ARV $\Delta_{\text {min }}$ depended (three-way anova on factors the muscle, side, and inter-electrode distance) on the inter-electrode distance $(F=8.93, P \ll 0.001)$, with $2.5 \mathrm{~mm}$ interelectrode distance resulting in larger $\Delta_{\min }$ than the other inter-electrode distances (SNK test, $P<0.001$ ).

\section{Optimal electrode location}

With the distances defined in the 'Materials and methods' section, the optimal electrode location was $23 \cdot 1 \pm 7 \cdot 7 \%$ of the mandibular angle-cantus distance, for the superficial masseter, and $24.4 \pm 6.6 \mathrm{~mm}$, for the anterior temporalis.

\section{EMG variables and inter-electrode distance}

Average rectified value and MNF were computed for the eight inter-electrode distances in the location resulting in $\Delta_{\min }$. Figure 3 reports the results. A threeway (factors: muscle, side, and inter-electrode distance) ANOVA of MNF revealed a dependency on the interelectrode distance $(F=9.02, P \ll 0.001)$. The post hoc SNK test disclosed pair-wise differences $(P<0.05)$ between MNF computed with inter-electrode distances which differed by at least $10 \mathrm{~mm}$.

A three-way (factors: muscle, side, and inter-electrode distance) ANOVA of ARV revealed a dependency on the muscle $(F=12.97, P<0.001)$ and inter-electrode distance $(F=88.34, P \ll 0.001)$. The anterior 

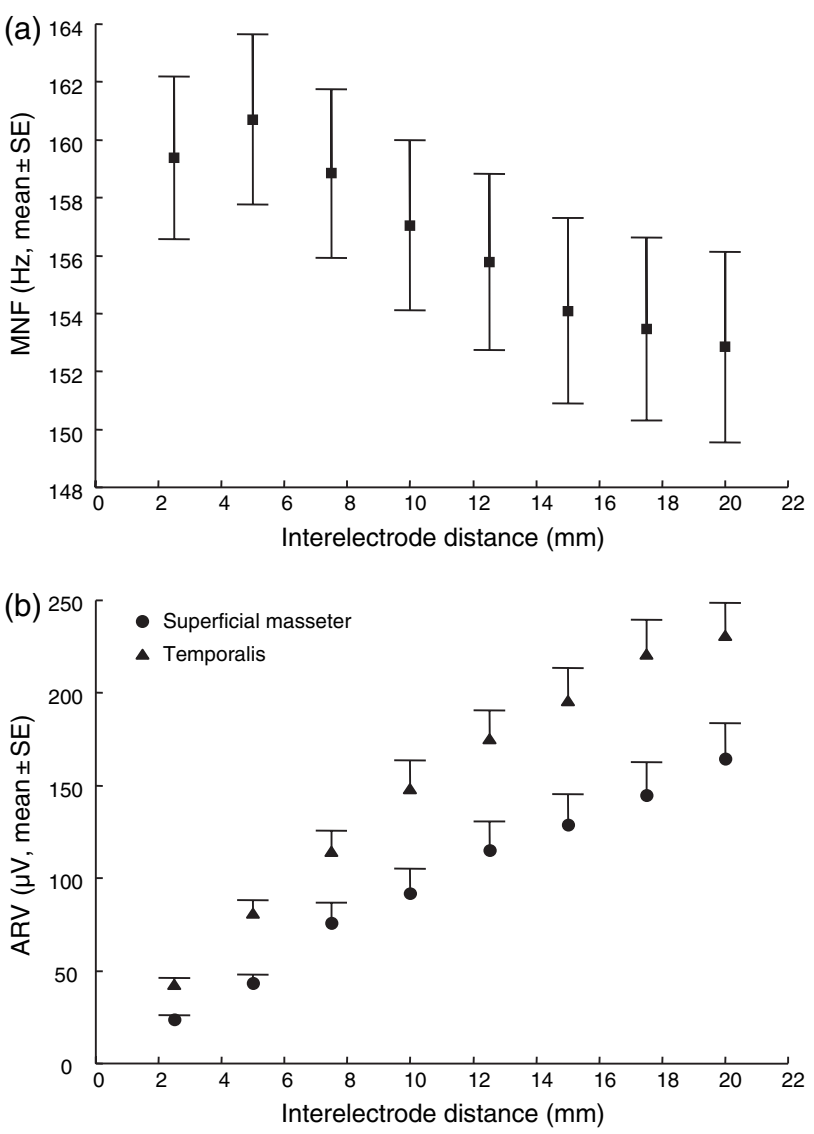

Fig. 3. (a) MNF values as a function of the inter-electrode distance. For each inter-electrode distance, the location leading to $\Delta_{\text {min }}$ has been selected. MNF values for the two muscles and both sides are pooled together as these factors do not affect the results (see text for the statistical analysis). (b) Same as (a) for ARV values. In this case the two muscles result in statistically different results (see text) and, thus, have been separated in reporting the results.

temporalis resulted in larger EMG amplitudes (Fig. 3) than the masseter muscle (SNK test, $P<0.001$ ). ARV values corresponding to different inter-electrode distances were significantly different $(P<0 \cdot 05)$.

\section{Discussion}

In this study we analysed the sensitivity of EMG variables in relation to electrode placement and interelectrode distance. Classic amplitude and spectral EMG variables have been considered as they have been used in many previous surface EMG studies for the assessment of jaw elevator muscles [see, for example Refs $(14,22-29)]$. The fibre length of the superficial masseter and anterior temporalis is approximately $25 \mathrm{~mm}$
(30), thus the end-plate and end-of-fiber components (2) constitute a major part of these signals. The sensitivity of EMG variables to electrode location was high when all the possible locations in the muscle portion investigated were compared (Fig. 2). Increasing the inter-electrode distance, the variability with electrode location significantly decreased, thus inter-electrode distances of at least $10-15 \mathrm{~mm}$ are suggested if the electrodes are placed without reference to optimisation criteria. Even with large inter-electrode distances, however, differences in EMG variable estimates from close locations over the muscle may be as large as $20-30 \%$. These values should correspond to the minimal differences which can be considered reliable when comparing subject groups or sets of measurements without using specific criteria for electrode placements. The sensitivity to electrode location found in this study on the jaw elevator muscles is similar to that observed in a previous study on the upper trapezius muscle (note, however, that in the latter case a larger skin area was covered) (21).

The variability of the estimates with electrode location probably also depends on the size of the electrodes. In this study we used small electrodes ( $1 \mathrm{~mm}$ diameter) in order to place them at a distance of $2.5 \mathrm{~mm}$, which allowed to compare results from a number of interelectrode distances. As the increase of electrode size may increase the detection volume, it is expected that the variability of the estimates reported in this study are larger than those obtained with larger electrodes. However, the dependency of the variability on interelectrode distance is not affected by this factor, thus the conclusions drawn in this study can be considered general. In addition, variability of amplitude estimates may be further decreased by normalization procedures (such as expressing the signal amplitude as a percentage of the amplitude recorded during a maximal voluntary contraction). While this approach may reduce the effect of some of the factors affecting absolute amplitude values, it may have other drawbacks, such as the poor reproducibility of the normalization contraction.

The minimal variability to electrode displacement of $\pm 2.5 \mathrm{~mm}$ is the percentage difference between EMG variables expected when a linear array with $2.5 \mathrm{~mm}$ inter-electrode distance (as that used in this study) is applied in different experimental sessions and when the optimal location defined in this study is used. The optimal location found in this study for the two muscles investigated varied considerably within subjects, which was 
probably because of the large spread of innervation zone locations and the short fibre length of these muscles (30).

Finally, we analysed which is the effect of the interelectrode distance on the EMG variable values. We observed, as expected (6), that MNF decreased and amplitude increased with increasing inter-electrode distance (as the detection volume increases with interelectrode distance) (Fig. 3). In particular, amplitude was significantly different for inter-electrode distances differing by as little as $2.5 \mathrm{~mm}$. Thus, inter-electrode distance is a critical parameter when results from different studies or subject groups are compared. When two adhesive electrodes with non-fixed inter-electrode distance are applied more than once, differences of the order of a few millimetres easily occur. This may introduce a variability in the results which may mask physiological or clinical observations. Thus, inter-electrode distance should be properly fixed in order to compare results among subjects, muscles, or experiments.

\section{Conclusions}

The main conclusions of this work are 1) care should be taken in interpreting results from EMG amplitude and spectral variables when the electrodes are placed without a criterion for electrode location; in this case, the sensitivity of EMG variable estimates to electrode displacements may be large (Fig. 2a); 2) increasing the inter-electrode distance determines a significant reduction of the variability of estimates with electrode displacement (Fig. 2a); and 3) spectral and amplitude EMG variables are very sensitive to inter-electrode distance (Fig. 3), which, thus, should be fixed when subjects or muscles are compared in the same or different experimental studies.

\section{Acknowledgments}

The authors are grateful to Roberto Bergamo for the help in performing the experimental measurements. This work was partially supported by Fondazione 'Cassa di Risparmio di Torino' and Compagnia di San Paolo, Torino, Italy.

\section{References}

1. Gydikov A, Gerilovsky L, Radicheva N, Trayanova N. Influence of the muscle fibre end geometry on the extracellular potentials. Biol Cybern. 1986;54:1-8.
2. Dimitrov GV, Dimitrova NA. Precise and fast calculation of the motor unit potentials detected by a point and rectangular plate electrode. Med Eng Phys. 1998;20:374-381.

3. Dimitrov GV, Disselhorst-Klug C, Dimitrova NA, Schulte E, Rau G. Simulation analysis of the ability of different types of multi-electrodes to increase selectivity of detection and to reduce cross-talk. J Electromyogr Kinesiol. 2003;13:125-138.

4. Dimitrova NA, Dimitrov GV, Nikitin OA. Neither high-pass filtering nor mathematical differentiation of the EMG signals can considerably reduce cross-talk. J Electromyogr Kinesiol. 2002;12:235-246.

5. Farina D, Merletti R, Nazzaro M, Caruso I. Effect of joint angle on EMG variables in muscles of the leg and thigh. IEEE Eng Med Biol. 2001;20:62-71.

6. Farina D, Cescon C, Merletti R. Influence of anatomical, physical and detection-system parameters on surface EMG. Biol Cybern. 2002;86:445-456.

7. Jensen C, Vasseljen O, Westgaard RH. The influence of electrode position on bipolar surface electromyogram recordings of the upper trapezius muscle. Eur J Appl Physiol. 1993;67:266-273.

8. Roy SH, De Luca CJ, Schneider J. Effects of electrode location on myoelectric conduction velocity and median frequency estimates. J Appl Physiol. 1986;61:1510-1517.

9. Zuniga N, Truong XT, Simons DG. Effects of skin electrode position on average electromyographic potentials. Arch Phys Med Rehabil. 1970;51:264-272.

10. Jankelson R, Pulley M. L'elettromiografia nella pratica odontoiatrica. Napoli: Beraha; 1985:13-16.

11. van der Glas HW, Lobbezoo F, Buchner R, van der Bilt A, Bosman F. Rate modulation of jaw elevator motor units as revealed from the low frequency power spectrum of the surface electromyogram in myogenous CMD patients. J Dent Res. 1994;5:1121-1128.

12. Hidaka $\mathrm{O}$, Iwasaki $\mathrm{M}$, Saito $\mathrm{M}$, Morimoto $\mathrm{T}$. Influence of clenching intensity on bite force balance, occlusal contact area, and average bite pressure. J Dent Res. 1999;7:1336-1344.

13. Gallo LM, Salis Gross SS, Palla S. Nocturnal masseter EMG activity of healthy subjects in a natural environment. J Dent Res. 1999;8:1436-1444.

14. Ferrario VF, Sforza C, Colombo A, Ciusa V. An electromyographic investigation of masticatory muscles symmetry in normo-occlusion subjects. J Oral Rehabil. 2000;27:33-40.

15. Iwasaki S, Tokunaga $T$, Baba S, Tanaka M, Kawazoe $T$. Noninvasive estimation of the location of the end plate in the human masseter muscle using surface electromyograms with an electrode array. J Osaka Dent Univ. 1990;24:135-140.

16. Tokunaga T, Baba S, Tanaka M, Kashiwagi K, Kimura K, Kawazoe T. Two-dimensional configuration of the myoneural junctions of human masticatory muscle detected with matrix electrode. J Oral Rehabil. 1998;25:329-334.

17. Bracco P, Vercellino V. Basic classification of 150 dysgnathic subjects according to Ricketts, Steiner and Cervera. Minerva Stomatol. 1980;29:1-38.

18. Bracco P, Deregibus A, Vercellino V. Comparative analysis using the computer 1: basal classification and antero-posterior positioning of the bases. Mondo Ortodontico. 1981;6:26-39. 
19. Masuda T, Miyano H, Sadoyama T. The position of innervation zones in the biceps brachii investigated by surface electromyography. IEEE Trans Biomed Eng. 1985;32:36-42.

20. Merletti R, Farina D, Gazzoni M. The linear electrode array: a useful tool with many applications. J Electromyogr Kinesiol. 2003;13:37-47.

21. Farina D, Merletti R. Comparison of algorithms for estimation of EMG variables during voluntary isometric contractions. J Electromyogr Kinesiol. 2000; 10:337-350.

22. Farina D, Madeleine P, Graven-Nielsen T, Merletti R, ArendtNielsen L. Stardardising surface electromyogram recordings for assessment of activity and fatigue in the human upper trapezius muscle. Eur J Appl Physiol. 2002;86:469-478.

23. Castroflorio T, Talpone F, Deregibus A, Piancino MG, Bracco P. Effect of a functional appliance on masticatory muscles of young adults suffering from muscle-related TMD. J Oral Rehabil. 2004;31:524-529.

24. Saifuddin M, Myamoto K, Ueda HM, Shikata N, Tanne K. An electromyographic evaluation of the bilateral symmetry and nature of masticatory muscle activity in jaw deformity patients during normal daily activities. J Oral Rehabil. 2003;30:578-586.

25. Ferrario VF, Sforza C, Tartaglia GM, Dellavia C. Immediate effect of a stabilization splint on masticatory muscle activity in temporomandibular disorders patients. J Oral Rehabil. 2002;29:810-815.

26. Scott BJ, Mason AG, Cadden SW. Voluntary and reflex control of the human temporalis muscle. J Oral Rehabil. 2002;29:634-643.

27. Kimoto K, Tamaki K, Yoshino T, Toyoda M, Celar AG. Correlation between elevator muscle activity and directionof sagittal closing pathway during unilateral chewing. J Oral Rehabil. 2002;29:430-434.

28. Tsai CM, Chou SL, Gale EN, McCall WD Jr. Human masticatory muscle activity and jaw position under experimental stress. J Oral Rehabil. 2002;29:44-51.

29. Pinho JC, Caldas EM, Mora MJ, Santana-Penin U. Electromyographic activity in patients with temporomandibular disorders. J Oral Rehabil. 2000;27:985-990.

30. van Eijden TMGJ, Korfage JAM, Brugman P. Architecture of the human jaw closing and jaw opening muscles. Anat Rec. 1997;248:464-474.

Correspondence: Tommaso Castroflorio, University of Torino, Department of Biomedical Sciences and Human Oncology, Specialization School of Orthodontics, C. so A.M. Dogliotti, 14 10100, Torino, Italy. E-mail: tcastroflorio@libero.it 